\title{
Biomarkers for risk of developing active tuberculosis in contacts of TB patients: a prospective cohort study
}

\author{
Niaina Rakotosamimanana ${ }^{1}$, Vincent Richard ${ }^{2,3}$, Vaomalala Raharimanga ${ }^{3}$, \\ Brigitte Gicquel $^{4}$, T. Mark Doherty ${ }^{5,6}$, Alimuddin Zumla ${ }^{7,8}$ and \\ Voahangy Rasolofo Razanamparany ${ }^{1}$
}

Affiliations: ${ }^{1}$ Unité des Mycobactéries, Institut Pasteur de Madagascar, Antananarivo, Madagascar. ${ }^{2}$ Unité d’Epidémiologie, Institut Pasteur de Madagascar, Antananarivo, Madagascar. ${ }^{3}$ Unité d'Epidémiologie, Institut Pasteur de Dakar, Dakar, Sénégal. ${ }^{4}$ Unité de Génétique Mycobactérienne, Institut Pasteur Paris, Paris, France. ${ }^{5}$ Staten Serum Institut, Copenhagen, Denmark. ${ }^{6}$ GlaxoSmithKline, Copenhagen, Denmark. ${ }^{7}$ Division of Infection and Immunity, University College London (UCL) and National Institute for Health Research Biomedical Research Centre, UCL Hospital, London, UK. ${ }^{8}$ The University of Zambia-University College London Medical School (UNZA-UCLMS) Research and Training Project, University Teaching Hospital, Lusaka, Zambia.

Correspondence: Niaina Rakotosamimanana, Institut Pasteur de Madagascar, 101 Antananarivo, Madagascar. E-mail: niainadpasteur.mg.

ABSTRACT Identifying those Mycobacterium tuberculosis latent-infected individuals most at risk of developing active tuberculosis (TB) using routine clinical and laboratory tests remains a huge challenge in TB control efforts. We conducted a prospective longitudinal study of clinical and laboratory markers associated with the risk of developing active TB in contacts with latent $M$. tuberculosis infection.

HIV-negative household contacts $(n=296)$ of pulmonary TB patients underwent monitoring of clinical features, full blood cell counts, tuberculin skin text (TST) and chest radiography performed regularly during 18 months of follow-up. Paired statistical tests, a Kaplan-Meier analysis and Cox proportional hazard modelling were performed on variables between contacts progressing or not progressing to active TB.

The appearance of TB disease symptoms in contacts was significantly associated with an elevated peripheral percentage of blood monocytes (adjusted hazard ratio (aHR) 6.25, 95\% CI 1.63-23.95; $\mathrm{p}<0.01$ ), $\mathrm{a} \geqslant 14 \mathrm{~mm}$ TST response (aHR 5.72, 95\% CI 1.22-26.80; $\mathrm{p}=0.03$ ) and an increased monocyte:lymphocyte ratio (aHR 4.97, 95\% CI 1.3-18.99; $\mathrm{p}=0.03$ ). Among contacts having TST $\geqslant 14 \mathrm{~mm}$, a strong association with risk of progression to TB was found with an elevated blood monocyte percentage (aHR 8.46, 95\% CI $1.74-41.22 ; \mathrm{p}<0.01)$.

Elevated percentage of peripheral blood monocytes plus an elevated TST response are potential biomarkers for identifying contacts of TB patients at highest risk of developing active TB.

@ERSpublications

Tuberculin skin tests combined with monocyte count improve evaluation of disease progression risk among TB contacts http://ow.ly/OtyWd

This article has supplementary material available from erj.ersjournals.com

Received: Feb 132015 | Accepted after revision: June 072015 | First published online: August 062015

Support statement: This study was supported by the Institut Pasteur de Madagascar core budget and was part of the VACSIS project, which was funded by the European Union, EU-INCO contract ICA4-CT-2002-10052. Funding information for this article has been deposited with FundRef.

Conflict of interest: None declared.

Copyright OERS 2015 


\section{Introduction}

Tuberculosis (TB) is an aerosol-borne infectious disease caused by Mycobacterium tuberculosis resulting in $\sim 8$ million incident $\mathrm{TB}$ cases and 1.5 million deaths annually $[1,2]$. However, most people with M. tuberculosis infection remain asymptomatic and develop what is known as latent $M$. tuberculosis infection (LTBI). The tuberculin skin test (TST) has traditionally been used to identify people with LTBI as they benefit from preventive TB treatment [3]. Approximately one-third of the world's population is thought to have LTBI. Identifying and treating all LTBI cases seems impractical as the disease is concentrated in countries with limited healthcare resources and because of the potential side-effects associated with chemotherapy [4]. Up to $10 \%$ of people with LTBI may develop active TB in the decades after infection [5]. The risk is highest in the years immediately following infection and in immunosuppressed individuals (e.g. people with HIV infection). Screening for the individuals most at risk of developing active TB from the huge "at-risk" reservoir of LTBI cases is an important goal of TB control efforts worldwide [6]. The prevalence of LTBI in close TB contacts is reported to be high: being a household contact is a serious risk factor associated with progression to active TB $[7,8]$.

Studies have suggested that the subsequent risk of developing disease in close contacts of TB patients was greatest among initially TST-sensitive individuals $[9,10]$. However, TST has known limitations, including cross-reactivity with bacillus Calmette-Guérin (BCG) vaccine and nontuberculous mycobacteria infections [11]. Furthermore, the TST and the more complex interferon- $\gamma$ release assays mostly identify all prior infections and are poor at predicting LTBI that will subsequently go on to develop active TB [12, 13]. Thus, there is a dire need for simple, sensitive laboratory biomarkers for risk of progression of M. tuberculosis infection to active disease [14].

The latent M. tuberculosis infection state is contained by an active immune response in the host initiated by the pathogen, permitting a controlled persistence of the organism. Several studies indicate that circulating immune cells are activated and recruited to the $M$. tuberculosis-infected lungs to form the granuloma where the $M$. tuberculosis proliferation is controlled by an active interaction of lymphocytes and infected macrophages [15-20]. In a previous study, we observed that peripheral white blood cell (WBC) subpopulation ratios varied according to TB clinical status in a limited number of BCG-vaccinated individuals from an area with a high TB burden [17]. More recently, observations from an HIV-positive or HIV-exposed population in South Africa also suggest an association between the ratio of circulating immune cells and the risk of TB disease [21, 22]. However, the hypothetical association of the peripheral WBC rates with risk of TB disease progression in HIV-negative at-risk populations requires confirmation. We conducted a prospective longitudinal cohort study to assess the role of peripheral blood WBC subpopulation counts as biomarkers of risk of progression to active disease in an HIV-negative population in a country with a high TB burden.

\section{Methods}

\section{Study setting and recruitment}

Close household contacts of patients with active pulmonary TB from Antananarivo (Madagascar) were investigated. The index cases of these household contacts were adults (aged >15 years) with newly diagnosed sputum smear positive and M. tuberculosis culture confirmed TB recruited from the main TB centre in Antananarivo. The inclusion criteria for the household contacts were age $>1$ year, living in the same house as the index case for $\geqslant 6$ months and asymptomatic for active TB. Asymptomatic was defined as an absence of clinical symptoms and signs characteristic of TB and a negative chest radiograph at entry to the study. Subjects who consented to an HIV test were enrolled and only HIV-negative individuals were included in the study. To investigate baseline responses for $M$. tuberculosis nonexposed individuals, ageand sex-matched community controls without symptoms or signs of TB and no known recent or sustained contact with TB cases were recruited at the post-exposure rabies centre of Institute Pasteur de Madagascar (Antananarivo, Madagascar).

Follow-up and monitoring of the household contacts was performed to detect TB symptoms for up to 18 months after inclusion: a clinical visit every 3 months for the first 6 months, and then a visit every 6 months for the remainder of the 18-month period. Any household contacts developing clinical symptoms suggestive of TB and/or a result of abnormal TST (TST highly positive $\geqslant 14 \mathrm{~mm}$ ) were referred to the anti-TB centre for additional investigations, including chest radiography. Individuals in whom the diagnosis of TB was confirmed during the study were recorded and treated as TB patients. For all subjects, epidemiological, clinical and bacteriological data were recorded prospectively on individual record forms.

\section{Sample size calculation}

The sample size calculation for the study was based on experience gained in an earlier study [23]. Briefly, for equal-sized groups of TB progressors and nonprogressors, it would be possible to detect a difference 
corresponding to a log report (average rate progressors/average nonprogressors) equal to 1.82 (consistent with the results of the study cited above) with a power of $90 \%$ and using an $\alpha$ error of 0.05 . For groups of uneven size (1 versus 4 for progressors versus nonprogressors), including 20 progressors and $80(20 \times 4)$ nonprogressors, the ratio of 1.82 would be detectable with a power of $98 \%$ (if $\alpha=0.05$ ). Applying this calculation to a power of $90 \%$, it would be possible to detect a ratio of 1.62 . In the proposed study in Madagascar, we wanted to include 100 index cases and expected (based on prior work) an average of five of six household contacts per index case.

\section{TST assays}

Study physicians performed the TST (10 units; Tuberculin Purified Protein Derivative, Sanofi Pasteur, Gentilly, France) at enrolment and after 3 months (M3) from inclusion. The results were read after $72 \mathrm{~h}$. Cutaneous induration $\geqslant 5 \mathrm{~mm}$ in diameter was considered to be a positive TST response and induration of $\geqslant 14 \mathrm{~mm}$ was considered to be highly reactive.

\section{Whole blood cell count}

Blood samples were drawn at inclusion and after 3 months. Blood samples were obtained from the index cases at inclusion and after completion of their TB treatment (12 months). Venous blood samples were drawn in EDTA anticoagulant Vacutainer tubes and stored at room temperature until the full blood cell (FBC) count was performed, using an ABX Pentra 120 Retic haematology analyser (Horiba ABX SAS, Les Ulis, France), according to the manufacturer's instructions. Absolute cell counts were expressed as cells per litre and percentage value per cell population (absolute cell count/total whole blood cell count $\times 100 \%$ ). The FBC count included information on red blood cells, platelets, total and differential WBC, which included neutrophils, eosinophils, basophils, monocytes and lymphocytes. A certified physician validated the formulas.

\section{Statistical analysis}

A comparison of the WBC subpopulations between the contacts that developed TB symptoms (symptomatic household contact (sHC)) and the household contacts that remained healthy (hHC) was firstly performed by paired sample analyses. Briefly, for every sHC, two age-matched hHCs were selected as controls for the paired analysis. Comparison using the Wilcoxon test for paired sample analysis was performed. The p-values were adjusted using the Bonferroni method to address multiple comparison concerns. Receiver operating characteristic (ROC) curves were then performed using the paired case-control to define the best FBC cut-off point for sensitivity and specificity. Risk of progression to active disease was estimated by performing survival analyses. The Kaplan-Meier method and the Gehan-Breslow-Wilcoxon test were used to compare the survival curves stratified by WBC rates. Cox proportional hazards models were generated to assess the association between WBC counts and development of active TB. Scaled Schoenfeld residuals were used to test the proportional hazards assumption of the Cox regression models and the Akaike information criteria (AIC) were compared between the models. Statistical tests were performed with R software (www.R-project. org). Tests were two sided and $\mathrm{p}<0.05$ was considered as significant.

\section{Ethical approval}

The study was approved by the national ethics committee of the Ministry of Health in Madagascar (authorisation number 038-SANPF/CAB). Participants were enrolled after appropriate counselling and explanation of the study. Only participants who had given their written informed consent were enrolled. Written informed consent was obtained from the legal guardians on the behalf of minors/child participants involved in the study.

\section{Results}

\section{Association of TST with risk of developing active TB in contacts}

296 HIV-negative TB household contacts of active TB index cases $(n=85)$ and 186 community controls were identified (table 1). Of these contacts, no samples were obtained from six subjects, three declined participation, one was a former $\mathrm{TB}$ case, while nine were lost to follow-up. BCG vaccination rate, evidenced by BCG scar, was $\sim 98.54 \%$ (table 1 ). The global sex ratio (male/female) was 0.71 .

At the end of the follow-up period, 12 (4.4\%) out of the remaining 289 household contacts developed symptoms consistent with active TB and were classified as sHC. The mean age of the sHC group was lower than that of the hHCs $(n=277, p=0.03$; table 1$)$. Nine $(75 \%)$ out of 12 sHC were children aged $<16$ years; however neither younger age nor sex was statistically associated with an elevated risk of developing active TB.

When comparing the proportion of TST-positive responders (cut-off TST $>5 \mathrm{~mm}$ ) no correlation was observed between the TST response and BCG vaccination status and there was no significant association between overall TST positivity and progression to active TB. However, it appeared that strong TST responders 
TABLE 1 Characteristics of the study cohorts

\begin{tabular}{lcccc} 
& Index cases & \multicolumn{2}{c}{ Household contacts } & Community controls \\
\cline { 2 - 4 } & & Symptomatic & Healthy & \\
\hline Subjects & 83 & 12 & 277 & 186 \\
Age years & $34.7(16-70)$ & $16.6(2-47)$ & $22.9(1-79)$ & $25.3(3-73)$ \\
Sex & & & & \\
$\quad$ Male & 45 & 5 & 125 & 89 \\
$\quad$ Female & 38 & 7 & 152 & 97 \\
BCG status & & & & $170(91.4)$ \\
Yes & $66(79.5)$ & $10(83.3)$ & $247(89.2)$ & 3 \\
No & 6 & 1 & 13 & 13 \\
Undetermined & 11 & 1 & 17 & $49(26.3)$ \\
TST response & $20(24.1)$ & $10(83.3)$ & $123(40.4)$ & $48(25.8)$ \\
$\geqslant 14$ mm & $18(21.7)$ & 0 & $89(32.1)$ & $89(47.9)$ \\
$5-14$ m & $7(8.4)$ & $2(16.7)$ & $63(22.7)$ & 0 \\
$<5$ mm & 38 & 0 & 2 & \\
Undetermined & & & & \\
\hline
\end{tabular}

Data are presented as n, mean (range) or $\mathrm{n}(\%)$. BCG: bacillus Calmette-Guérin; TST: tuberculin skin test.

were at higher risk of subsequently developing active TB. 10 (83\%) out of the 12 sHCs had a TST induration of $\geqslant 14 \mathrm{~mm}$ at enrolment (table 1 ). A TST $\geqslant 14 \mathrm{~mm}$ was associated with a significant risk of progression to active disease in household contacts (age-adjusted hazard ratio 5.47, 95\% CI 1.18-25.33; $\mathrm{p}=0.03$ ).

\section{Peripheral blood monocyte counts were significantly different between household contacts with different TB outcomes during follow-up}

Figure 1a depicts the peripheral leukocyte count at inclusion in all study groups. The subjects with known exposure to M. tuberculosis (index cases and household contacts) showed a significantly higher level of leukocytes $(\mathrm{p}<0.05)$ than community controls. However, when the WBC counts were stratified into blood cell subpopulations, significant differences according to clinical status were observed.

A severe decrease in the lymphocyte count $(p<0.001)$ (fig. 1c) associated with an increase of both neutrophil $(p<0.001)$ (fig. $1 b)$ and monocyte rates $(p<0.05)$ (fig. $1 \mathrm{~d})$ was globally observed in those study participants with active disease compared to the asymptomatic individuals. Furthermore, an elevated monocyte count was observed in the TB contacts compared to the community controls $(\mathrm{p}<0.001)$ (fig. 1c). Those contacts that subsequently developed TB displayed a WBC pattern distinct from both the index cases and the other healthy groups (community controls and hHC). The sHC study participants had significantly elevated monocyte counts compared to both the community controls and hHC groups ( $\mathrm{p}<0.001)$ (fig. 1c).

Moreover, among those individuals with a TST $\geqslant 14 \mathrm{~mm}$, the $\mathrm{sHC}$ group segregated from the healthy (community controls and hHC) individuals with regards to lymphocyte and neutrophil counts, and segregated from the TB patients with regards to monocyte counts (online supplementary material). Peripheral WBC populations that segregated the sHC $(n=12)$ from the hHC $(n=24)$ paired sample analyses (table 2) showed that both the monocyte and lymphocyte absolute counts were significantly different in the hHC when compared to $\mathrm{sHC}$ ( $\mathrm{p}=0.04$ and $\mathrm{p}=0.02$, respectively). However, after Bonferroni adjustment, the monocyte percentage was the only significant difference between sHC and hHC $(\mathrm{p}<0.01)$ (table 2$)$.

\section{Peripheral WBC counts in TB patients after successful anti-TB treatment}

To assess whether the variations observed in the peripheral WBCs were associated with active TB, we also measured WBC counts before and after treatment for TB in the index cases. Consistent with this hypothesis, the total WBC count decreased post-treatment $(\mathrm{p}<0.01)$ (fig. 2). In particular, the neutrophil and monocyte fractions in these treated patients were significantly reduced upon completion of treatment when compared to their rates at enrolment (fig. 2). While lymphocyte counts increased slightly, this was not significant in absolute terms (data not shown), but the decline in other cell types meant that the increase was highly significant $(\mathrm{p}<0.001)$ (fig. 2$)$ as a percentage of total WBCs. Thus, post-treatment, the index cases showed a pattern (decreasing monocytes and neutrophils and increasing lymphocytes) that suggested that they were moving towards a profile similar to that observed in healthy individuals. 
a)

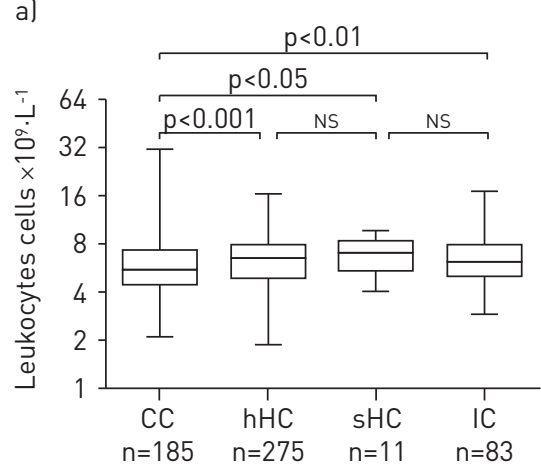

Clinical status

c)

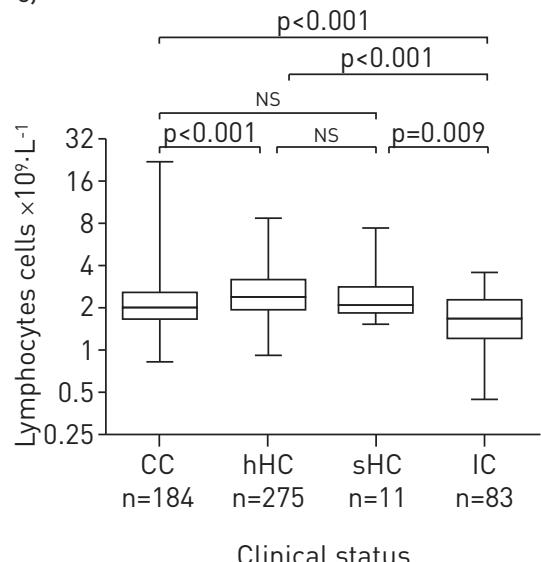

b) $\quad \mathrm{p}<0.001$

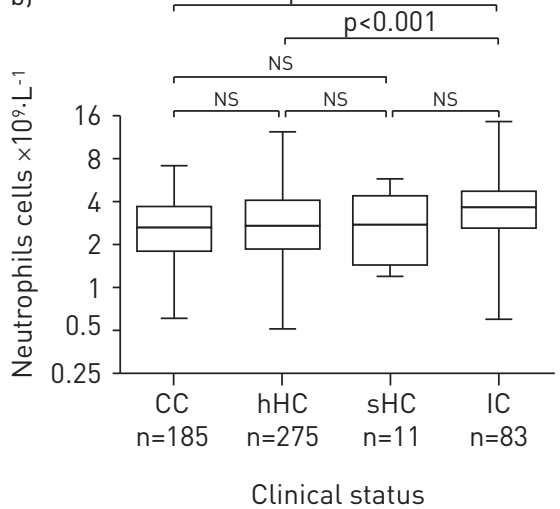

d)
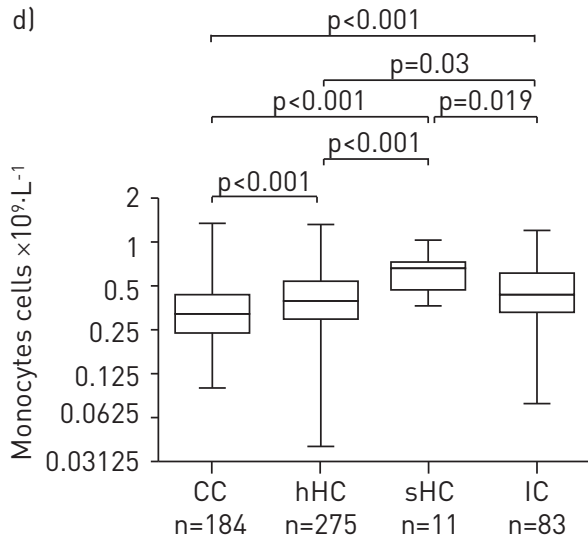

Clinical status

FIGURE 1 Comparison of white blood cell count in the different clinical status groups prior to inclusion in the study. al Leukocytes; b) neutrophils; cl lymphocytes; d) monocytes. Data are presented as median and ranges of absolute cell counts. CC: community controls; hHC: household contacts that remained healthy; $\mathrm{sHC}$ : household contacts that developed tuberculosis (TB) symptoms in the course of follow-up; IC: TB index cases; NS: nonsignificant. Mann-Whitney U-tests were used for the pairwise comparison of groups.

Assessment of risk of developing active TB according to peripheral blood monocyte rate and TST As the monocyte percentage was the biomarker that showed most significant difference between sHC and hHC (table 2), a ROC analysis was performed to identify a cut-off associated with elevated risk for developing $\mathrm{TB}$ in the $\mathrm{TB}$ contacts. A cut-off point from the ROC curves of $7.5 \%$ monocytes in total

TABLE 2 Descriptive paired analysis of cohorts with regard to the development of tuberculosis symptoms

\begin{tabular}{lcccc} 
& sHC & hHC & p-value & Bonferroni correction \\
\hline $\begin{array}{l}\text { Subjects n } \\
\text { Leukocytes }\end{array}$ & 12 & 24 & & \\
$\begin{array}{l}\text { Neutrophils } \\
\text { cells } \times 109 \cdot \mathrm{L}^{-1}\end{array}$ & $7.3(4.1-9.8)$ & $7.9(3.2-14.9)$ & 0.39 & $\mathrm{NS}$ \\
$\quad \begin{array}{l}\text { Percentage } \\
\text { Lymphocytes }\end{array}$ & $46.1(1.2-5.9)$ & $3.8(0.8-10.2)$ & 0.42 & $\mathrm{~N}$ \\
$\quad$ cells $\times 109 \cdot \mathrm{L}^{-1}$ & $2.2(1.5-7.4)$ & $2.85(1.3-8.4)$ & $0.02^{\#}$ & $\mathrm{~N}$ \\
$\quad \begin{array}{l}\text { Percentage } \\
\text { Monocytes }\end{array}$ & $37.6(18.5-77.5)$ & $37.8(18.5-74.2)$ & 0.47 & 0.14 \\
$\quad$ cells $\times 109 \cdot \mathrm{L}^{-1}$ & $0.68(0.38-1.04)$ & $0.47(0.19-0.86)$ & $0.04^{\#}$ & $\mathrm{NS}$ \\
$\quad$ Percentage & $8.5(6.4-16.5)$ & $6.1(2.7-12.5)$ & $<0.001^{\#}$ & 0.28 \\
\end{tabular}

Data are presented as median (range), unless otherwise stated. sHC: symptomatic household contacts; hHC: healthy household contacts; NS: nonsignificant. ${ }^{\#}$ : statistically significant values. 


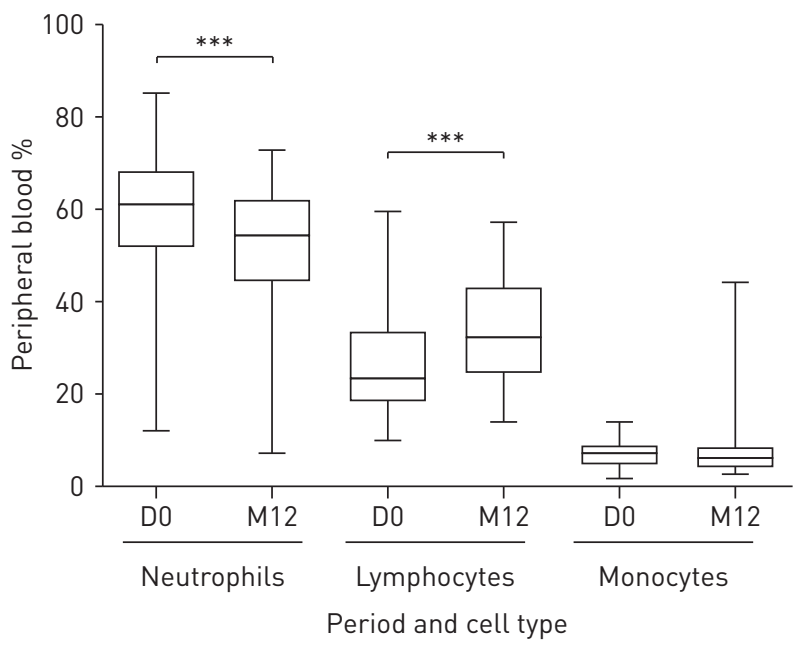

FIGURE 2 Comparison of white
blood cell rates in the tuberculosis
patients. Data are presented as
median and ranges of cell
percentages. D0: at inclusion ( $n=83$ );
M12: after treatment $(n=66)$. Mann-
Whitney U-tests were used for the
pairwise comparison of groups. ${ }^{* * *}$ :
p $<0.001$.

peripheral blood mononuclear cells gave the best separation, and was associated with a sensitivity and specificity of $75 \%$ (fig. 3 ).

TST $\geqslant 14 \mathrm{~mm}$ and blood monocyte rate $\geqslant 7.5 \%$ in the TB contacts remained associated with the highest risk of developing active TB adjusted for lymphocyte count (table 3). Household contacts with both TST $\geqslant 14 \mathrm{~mm}$ and monocyte rate $\geqslant 7.5 \%$ were associated with a significantly elevated risk of progressing to active TB ( $\mathrm{p}<0.001$ by the log-rank test) (fig. 4). The hazard ratio (HR) for developing TB symptoms in household contacts with TST $\geqslant 14 \mathrm{~mm}$, monocyte:lymphocyte ratio and peripheral blood monocyte rate $\geqslant 7.5 \%$ were significantly high (table 3). To address the potential confounding of age and sex, these covariates were included in Cox models. After adjustment for age, sex and blood lymphocyte count, household contacts with both TST $\geqslant 14 \mathrm{~mm}$ and peripheral blood monocyte rate $\geqslant 7.5 \%$ remained significantly at risk of developing active TB (HR 8.46, 95\% CI 1.73-41.22; p<0.01), with the lowest AIC (table 3).

\section{Discussion}

Developing tests to determine which individuals with LTBI are at the greatest risk of progressing to active $\mathrm{TB}$ would allow the identification and treatment of at-risk individuals and reduce the number of active TB cases. This would be a major step forward for TB control programmes. We conducted this longitudinal cohort study to examine the association of peripheral WBC counts with the risk of TB in HIV-negative TB close household contacts. Our study shows the following findings.

Consistent with other studies [24-26] we confirmed that the prognostic power of a positive TST alone in predicting LTBI progression to active TB disease is low. As TB is endemic in Madagascar and the coverage rate is high for BCG vaccination, a weak TST response may not be specific for the detection of M. tuberculosis infection, and it is to be expected that the healthy groups are heterogeneous; potentially

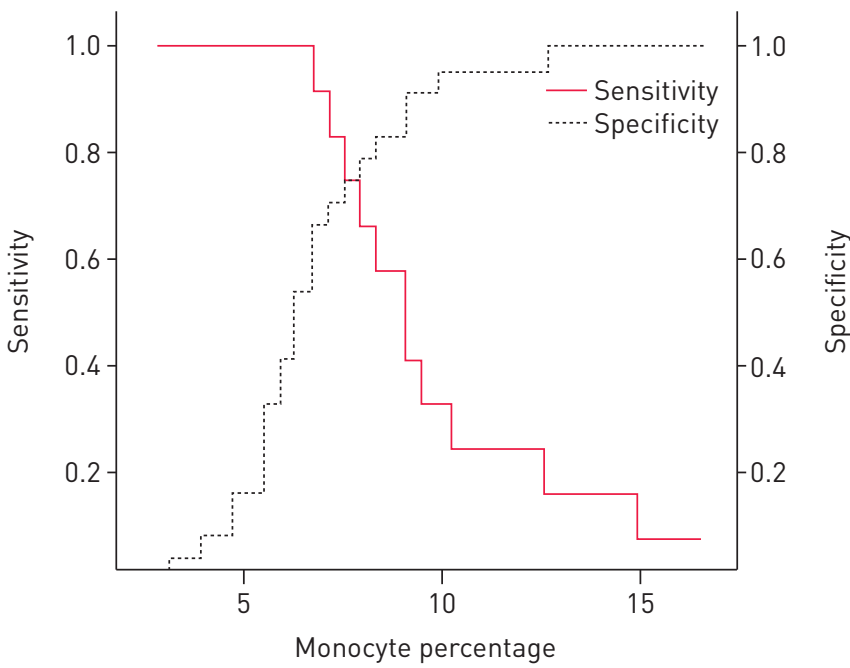

FIGURE 3 Specificity and sensitivity curves according to blood monocyte percentages in the tuberculosis contact population. A cut-off point of $7.5 \%$ was obtained. 
TABLE 3 Parameters analysed to predict risk of progression to active tuberculosis in household contacts

\begin{tabular}{|c|c|c|c|c|c|}
\hline & $\begin{array}{c}\text { Crude } \\
\text { HR }(95 \% \text { CI) }\end{array}$ & p-value & $\begin{array}{c}\text { Adjusted } \\
\text { HR }(95 \% \text { CI) }\end{array}$ & p-value & AIC \\
\hline Age & $0.97(0.92-1.02)$ & 0.23 & $0.98(1.02-1.03)$ & 0.93 & \\
\hline Lymphocytes & $0.99(0.94-1.04)$ & 0.66 & $0.97(0.53-1.79)$ & 0.94 & \\
\hline $\mathrm{TST} \geqslant 14 \mathrm{~mm}$ & $5.5(1.2-25.3)$ & $0.03^{\#}$ & $5.72(1.22-26.80)$ & $0.03^{\#}$ & \\
\hline Monocytes $\geqslant 7.5 \%$ & $6.08(1.61-22.91)$ & $<0.01^{\#}$ & $6.25(1.63-23.95)$ & $<0.01^{\#}$ & 108.73 \\
\hline Monocyte/lymphocyte ratio & $4.49(1.19-16.94)$ & & $4.97(1.30-18.99)^{9}$ & $0.02^{\#}$ & 113.39 \\
\hline Monocytes $\geqslant 7.5 \%+$ TST $\geqslant 14 \mathrm{~mm}$ & $8.78(1.82-42.32)$ & $<0.01^{\#}$ & $8.46(1.73-41.22)^{+}$ & $<0.01^{\#}$ & 80.81 \\
\hline
\end{tabular}

$\mathrm{n}=274$. HR: hazard ratio; AIC: Akaike information criterion per Cox model; TST: tuberculin skin test. \#: statistically significant; ${ }^{\text {ๆ: }}$ adjusted for age, sex and TST $\geqslant 14 \mathrm{~mm}^{+}{ }^{*}$ : adjusted for age, sex and lymphocyte count.

containing both latently infected and uninfected individuals. We therefore used the more stringent definition that a strong TST response with an induration in the TST of $\geqslant 14 \mathrm{~mm}$ equated to LTBI [27]. Using this definition, it was also found that a strong TST response $(\geqslant 14 \mathrm{~mm})$ was associated with a significantly elevated risk for subsequent progress to TB among contacts, as described previously [28].

Household contacts with LTBI aged $<16$ years appear to be at slightly higher risk of progressing to active TB in this population than adults. This is in contrast to observations from countries with a low TB burden where the older age group was found to be at higher risk of developing TB [29]. However, this may reflect the different age structure of our cohort, which had fewer elderly individuals, a common pattern in the age structures of developing countries, or different patterns of transmission. Our data suggest that it may be worth extending the World Health Organization recommendation for TB preventive treatment to individuals aged from $<5$ to $<16$ years in countries that have a high TB burden and populations of similar age distributions to that studied here.

An increased risk of subsequently developing TB was associated with elevated peripheral blood monocyte rate and TST response $\geqslant 14 \mathrm{~mm}$ among TB household contacts in this study. Differences in the peripheral blood cell count have been shown to identify elevated risk of developing active TB in HIV-infected individuals $[13,30]$. However, while the monocyte:lymphocyte ratio seemed to give the best predictive value in the HIV-infected individuals, observations from the present study in HIV-negative TB contacts suggested that the peripheral blood monocyte rate alone can identify elevated risk of progression to active TB, and when combined with a strong TST response gave a better risk association than the monocyte: lymphocyte ratio according to the AIC obtained from the different models. The effect of HIV on

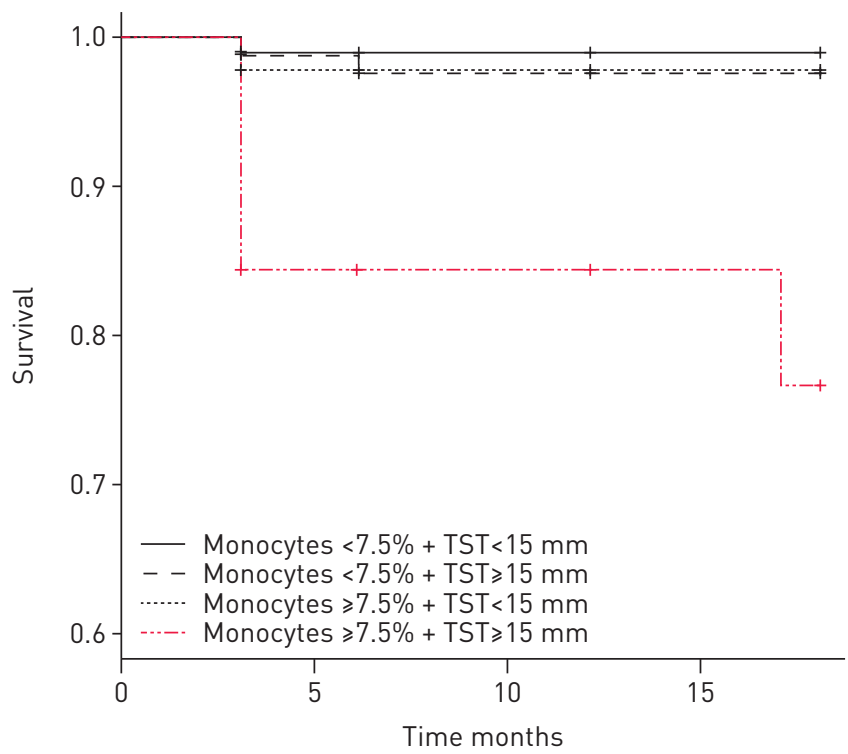

FIGURE 4 Kaplan-Meier estimates for overall survival from diagnosis of active tuberculosis (TB) symptoms in the TB contacts. The groups were divided based upon peripheral monocyte percentages and tuberculin skin testing. 
lymphocyte and leukocyte numbers [31] could explain the differences observed in these HIV-positive and -negative populations.

Monocytes are produced in the bone marrow from monoblasts and then circulate in the bloodstream and reside in the spleen. The circulating monocytes are precursors for tissue macrophages that are implicated in defence against a range of microbial pathogens $[32,33]$. These cells are actively recruited from the bloodstream to form the lung granuloma that contain $M$. tuberculosis infection, but it has also been established that $M$. tuberculosis can inhibit the host immune response by multiple mechanisms, in particular by inhibiting the phagocytic process and using the phagocytes as ecological niches where the pathogen can replicate [32-35]. Chemokines such as monocyte chemoattractant protein-1 are suggested to be involved in the massive monocyte recruitment to the lung to control M. tuberculosis infection [36, 37]. A role for type I interferons in the accumulation of myeloid cell populations in the lung and pulmonary recruitment of inflammatory monocytes that lead to TB disease immunopathology has also been suggested [38-40]. An ongoing infection with an inadequate adaptive immune response against the pathogen may explain the increased percentage of blood monocytes observed in the TB contacts in our study prior to the development of active disease.

It has already been reported that patients with active TB have an increased frequency of peripheral blood monocytes compared to healthy individuals, and this study and others have shown that effective anti-TB chemotherapy can reverse this $[17,41,42]$. The high count of peripheral monocytes also observed in the $\mathrm{TB}$ contacts prior to progression to an active TB in the present study is consistent with the hypothesis that this could be a biomarker for progressive TB [42, 43], and our study suggests that this is the case even before the appearance of symptoms.

In summary, combining TST, a technique widely used to assess an M. tuberculosis infection in developing countries, and the peripheral blood monocyte count, a technique available in many health centres, may serve as a simple, cheap and practical test to identify those most at risk of progression to TB disease. If confirmed in larger studies and in diverse populations, using both tests in TB contacts could avoid unnecessary treatment and may improve the identification of individuals that need to be prioritised for treatment.

\section{Acknowledgements}

We thank Soa Fy Andriamandimby (Institut Pasteur de Madagascar, Antananarivo, Madagascar) for the recruitment of the participants, the Centre de Biologie Clinique of the Institut Pasteur de Madagascar for blood tests, the clinical physicians of the Dispensaire Anti-Tuberculeux d'Antananarivo, the Radiology Department of the Institut d'Hygiène Sociale, the staff of the National Mycobacterial Laboratory of the Ministry of Health and the National TB Control Programme of the Ministry of Health (all Antananarivo, Madagascar) for their contribution to the study and Christophe Rogier (Institut Pasteur de Madagascar) for fruitful discussions. A. Zumla acknowledges support from the European and Developing Countries Clinical Trials Partnership, National Institutes of Health Research University College London Hospitals Biomedical Research Centre, and the European Union.

\section{References}

WHO. World Health Organisation Global Tuberculosis Report 2014. Geneva, WHO, 2014.

2 WHO. Guidance for National Tuberculosis Programmes on the Management of Tuberculosis in Children. WHO/ HTM/TB/2006.371 2006. Geneva, WHO, 2006.

3 Stagg HR, Zenner D, Harris RJ, et al. Treatment of latent tuberculosis infection: a network meta-analysis. Ann Intern Med 2014; 161: 419-428.

4 Zumla A, Raviglione M, Hafner R, et al. Tuberculosis. N Engl J Med 2013; 368: 745-755.

5 Andrews JR, Noubary F, Walensky RP, et al. Risk of progression to active tuberculosis following reinfection with Mycobacterium tuberculosis. Clin Infect Dis 2012; 54: 784-791.

6 Beamer GL, Flaherty DK, Vesosky B, et al. Peripheral blood gamma interferon release assays predict lung responses and Mycobacterium tuberculosis disease outcome in mice. Clin Vaccine Immunol 2008; 15: 474-483.

7 Fox GJ, Barry SE, Britton WJ, et al. Contact investigation for tuberculosis: a systematic review and meta-analysis. Eur Respir J 2014; 41: 140-156.

8 Morán-Mendoza O, Marion SA, Elwood K, et al. Risk factors for developing tuberculosis: a 12-year follow-up of contacts of tuberculosis cases. Int J Tuberc Lung Dis 2010; 14: 1112-1119.

9 Diel R, Loddenkemper R, Meywald-Walter K, et al. Predictive value of a whole blood IFN- $\gamma$ assay for the development of active tuberculosis disease after recent infection with Mycobacterium tuberculosis. Am J Respir Crit Care Med 2008; 177: 1164-1170.

10 Diel R, Loddenkemper R, Niemann S, et al. Negative and positive predictive value of a whole-blood interferon- $\gamma$ release assay for developing active tuberculosis: an update. Am J Respir Crit Care Med 2011; 183: 88-95.

11 Menzies D. What does tuberculin reactivity after bacille Calmette-Guérin vaccination tell us? Clin Infect Dis 2000; 31: Suppl 3, S71-S74.

12 Andersen P, Munk ME, Pollock JM, et al. Specific immune-based diagnosis of tuberculosis. Lancet 2000; 356 : 1099-1104.

13 Diel R, Loddenkemper R, Nienhaus A. Predictive value of interferon- $\gamma$ release assays and tuberculin skin testing for progression from latent TB infection to disease state: a meta-analysis. Chest 2012; 142: 63-75.

14 Wallis RS, Pai M, Menzies D, et al. Biomarkers and diagnostics for tuberculosis: progress, needs, and translation into practice. Lancet 2010; 375: 1920-1937. 
15 Zuñiga J, Torres-García D, Santos-Mendoza T, et al. Cellular and humoral mechanisms involved in the control of tuberculosis. Clin Dev Immunol 2012; 2012: 193923.

16 Berry MP, Graham CM, McNab FW, et al. An interferon-inducible neutrophil-driven blood transcriptional signature in human tuberculosis. Nature 2010; 466: 973-977.

17 Rakotosamimanana N, Doherty TM, Andriamihantasoa LH, et al. Expression of TNF-alpha-dependent apoptosis-related genes in the peripheral blood of Malagasy subjects with tuberculosis. PLoS One 2013; 8: e61154.

18 Dhanasekaran S, Jenum S, Stavrum R, et al. Identification of biomarkers for Mycobacterium tuberculosis infection and disease in BCG-vaccinated young children in Southern India. Genes Immun 2013; 14: 356-364.

19 Druszczynska M, Wlodarczyk M, Janiszewska-Drobinska B, et al. Monocyte signal transduction receptors in active and latent tuberculosis. Clin Dev Immunol 2013; 2013: 851452.

20 Tran HT, Van den Bergh R, Vu TN, et al. The role of monocytes in the development of tuberculosis-associated immune reconstitution inflammatory syndrome. Immunobiology 2014; 219: 37-44.

21 Naranbhai V, Moodley D, Chipato T, et al. The association between the ratio of monocytes: lymphocytes and risk of tuberculosis among HIV-infected postpartum women. J Acquir Immune Defic Syndr 2014; 67: 573-575.

22 Naranbhai V, Hill AV, Abdool Karim SS, et al. Ratio of monocytes to lymphocytes in peripheral blood identifies adults at risk of incident tuberculosis among HIV-infected adults initiating antiretroviral therapy. J Infect Dis 2014; 209: 500-509.

23 Lienhardt C, Bennett S, Del Prete G, et al. Investigation of environmental and host-related risk factors for tuberculosis in Africa. I. Methodological aspects of a combined design. Am J Epidemiol 2002; 155: 1066-1073.

24 Wallis RS, Kim P, Cole S, et al. Tuberculosis biomarkers discovery: developments, needs, and challenges. Lancet Infect Dis 2013; 13: 362-372.

25 Abubakar I, Stagg HR, Whitworth $\mathrm{H}$, et al. How should I interpret an interferon gamma release assay result for tuberculosis infection? Thorax 2013; 68: 298-301.

26 Kik SV, Franken WP, Mensen M, et al. Predictive value for progression to tuberculosis by IGRA and TST in immigrant contacts. Eur Respir J 2010; 35: 1346-1353.

27 Gallant CJ, Cobat A, Simkin L, et al. Tuberculin skin test and in vitro assays provide complementary measures of antimycobacterial immunity in children and adolescents. Chest 2010; 137: 1071-1077.

28 Wisnivesky JP, Kaplan J, Henschke C, et al. Evaluation of clinical parameters to predict Mycobacterium tuberculosis in inpatients. Arch Intern Med 2000; 160: 2471-2476.

29 Mendes MA, Gaio R, Reis R, et al. Contact screening in tuberculosis: can we identify those with higher risk? Eur Respir J 2013; 41: 758-760.

30 Naranbhai V, Kim S, Fletcher $\mathrm{H}$, et al. The association between the ratio of monocytes:lymphocytes at age 3 months and risk of tuberculosis (TB) in the first two years of life. BMC Med 2014; 12: 120.

31 Hasegawa A, Liu H, Ling B, et al. The level of monocyte turnover predicts disease progression in the macaque model of AIDS. Blood 2009; 114: 2917-2925.

32 Soehnlein O, Zernecke A, Eriksson EE, et al. Neutrophil secretion products pave the way for inflammatory monocytes. Blood 2008; 112: 1461-1471.

33 Serbina NV, Jia T, Hohl TM, et al. Monocyte-mediated defense against microbial pathogens. Annu Rev Immunol 2008; 26: 421-452.

34 Srivastava S, Ernst JD, Desvignes L. Beyond macrophages: the diversity of mononuclear cells in tuberculosis Immunol Rev 2014; 262: 179-192.

35 Armstrong JA, Hart PD. Response of cultured macrophages to Mycobacterium tuberculosis, with observations on fusion of lysosomes with phagosomes. J Exp Med 1971; 134: 713-740.

36 Kim K, Sohn H, Kim JS, et al. Mycobacterium tuberculosis Rv0652 stimulates production of tumour necrosis factor and monocytes chemoattractant protein-1 in macrophages through the Toll-like receptor 4 pathway. Immunology 2012; 136: 231-240

37 Arias MA, Jaramillo G, López YP, et al. Mycobacterium tuberculosis antigens specifically modulate CCR2 and MCP-1/CCL2 on lymphoid cells from human pulmonary hilar lymph nodes. J Immunol 2007; 179: 8381-8391.

38 Dorhoi A, Yeremeev V, Nouailles G, et al. Type I IFN signaling triggers immunopathology in tuberculosissusceptible mice by modulating lung phagocyte dynamics. Eur J Immunol 2014; 44: 2380-2393.

39 Antonelli LR, Gigliotti Rothfuchs A, Goncalves R, et al. Intranasal poly-IC treatment exacerbates tuberculosis in mice through the pulmonary recruitment of a pathogen-permissive monocyte/macrophage population. $J$ Clin Invest 2010; 120: 1674-1682.

40 Abebe M, Doherty TM, Wassie L, et al. Expression of apoptosis-related genes in an Ethiopian cohort study correlates with tuberculosis clinical status. Eur J Immunol 2010; 40: 291-301.

41 Sánchez MD, García Y, Montes C, et al. Functional and phenotypic changes in monocytes from patients with tuberculosis are reversed with treatment. Microbes Infect 2006; 8: 2492-2500.

42 Liu Y, Jiang J, Wang X, et al. miR-582-5p is upregulated in patients with active tuberculosis and inhibits apoptosis of monocytes by targeting FOXO1. PLoS One 2013; 8: e78381.

43 Juffermans NP, Verbon A, van Deventer SJ, et al. Elevated chemokine concentrations in sera of human immunodeficiency virus (HIV)-seropositive and HIV-seronegative patients with tuberculosis: a possible role for mycobacterial lipoarabinomannan. Infect Immun 1999; 67: 4295-4297. 